\title{
Averting cracks caused by insertion reaction in lithium-ion batteries
}

Yuhang $\mathrm{Hu}$, Xuanhe Zhao and Zhigang Suo a

School of Engineering and Applied Sciences, Harvard University, Cambridge, MA 02138

\begin{abstract}
In a lithium-ion battery, both electrodes are atomic frameworks that host mobile lithium ions. When the battery is being charged or discharged, lithium ions diffuse from one electrode to the other. Such an insertion reaction deforms the electrodes, and may cause the electrodes to crack. This paper uses fracture mechanics to determine the critical conditions to avert cracking. The method is applied to cracks induced by the mismatch between phases in crystalline particles of $\mathrm{LiFePO}_{4}$.
\end{abstract}

a email: suo@seas.harvard.edu 
Lithium-ion batteries of high energy densities have rapidly become the batteries of choice for portable electronics. ${ }^{1}$ These batteries are being further developed as a technology for clean and secure energy. ${ }^{2-4}$ Battery-powered cars will reduce pollution, promote renewable energy, and maintain individual mobility.

At the heart of a lithium-ion battery is a process that couples electrochemistry and mechanics. In the battery, two electrodes are separated by an electrolyte. Each electrode is an atomic framework that conducts both lithium ions and electrons, while the electrolyte conducts lithium ions but not electrons. The two electrodes are kept in the open-circuit condition when the battery is in storage, but are connected by an electronic conductor when the battery is being charged or discharged. During charging or discharging, the difference in the electrochemical potentials of lithium in the two electrodes motivates lithium ions to move from one electrode to the other through the electrolyte. To maintain electrical neutrality, electrons flow from one electrode to the other through the electronic conductor. This process, in which an atomic framework absorbs or releases mobile atoms, is known as an insertion reaction. ${ }^{5,6}$ When large amounts of lithium atoms are inserted into or extracted from the framework, the electrodes deform substantially.

The insertion-induced deformation is often constrained by the mismatch between active and inactive materials, between grains of different crystalline orientations, and between phases of different concentrations of lithium (Fig.1). Under such constraints, the insertion or extraction of lithium induces in an electrode a field of stress, which may cause the electrode to form cracks. ${ }^{7-9}$ The cracks may break pieces of active material from the electrode, causing the capacity of the battery to fade.9,10 The cracks also expose fresh surfaces of active materials to the electrolyte, possibly degrading the chemical stability and safety of the battery. ${ }^{10}$

This paper describes a method to determine conditions that avert insertion-induced cracking. To focus on essential ideas, we consider cracks caused by the mismatch between different phases in LiFePO4, a material used as cathodes in lithium-ion batteries due to its high 
energy density, low cost, and thermal stability. ${ }^{18}$ For such a cathode, particles of LiFePO4 are embedded in a compliant matrix. Although LiFePO4 has low electronic conductivity in its native state, electrochemical performance can be improved substantially in many ways, such as carbon coating ${ }^{19}$, doping ${ }^{20,21}$ and reducing particle size ${ }^{22,23}$. Our calculation determines the critical size of particles, below which cracking will not occur. This conclusion is consistent with existing experimental observations. ${ }^{11,12}$

Insertion-induced cracking in batteries have been analyzed in previous studies, using thin-film model ${ }^{13}$ and core-shell model ${ }^{14-17}$. Here we describe a model on the basis of the geometry of LiFePO4 particles. Our model is formulated in terms of fracture mechanics. ${ }^{24}$ Crack-like flaws are assumed to pre-exist in a crystalline particle of LiFePO4. The particle is in a state of stress caused by the mismatch between two phases of the crystal. We ask if the stress will cause any of the flaws to advance. The elastic energy in the particle reduces when a crack advances. The reduction of the elastic energy in the particle associated with the crack advancing a unit area defines the energy release rate G. Dimensional considerations dictate that the energy release rate should take the form

$$
\mathrm{G}=\mathrm{ZE} \varepsilon_{\mathrm{m}}^{2} \mathrm{~d}
$$

where $\mathrm{E}$ is an elastic modulus, $\varepsilon_{\mathrm{m}}$ a mismatch strain, and $\mathrm{d}$ a length characteristic of the size of the particle. The dimensionless coefficient $\mathrm{Z}$ is determined by solving the boundary-value problem of elasticity.

As the crack advances in the particle, the elastic energy of the particle reduces, but the area of the crack increases. The crack cannot advance if

$$
\mathrm{G}<2 \gamma
$$

where $\gamma$ is the surface energy.

One difficulty in applying fracture mechanics is that the dimensionless coefficient $\mathrm{Z}$ varies with a large number of parameters, including ratios of various elastic moduli, of various 
mismatch strains, and of various lengths. To circumvent this difficulty, we adopt the following approach. We will study particles of shapes observed in experiments, and use the anisotropic elastic moduli of LiFePO4. Once the particle is given, $\mathrm{Z}$ can still vary with the location and length of the crack. We will fix the location of the crack on the cleavage planes of the crystal, and then vary the length of the crack to maximize $\mathrm{Z}$. When all parameters are fixed except for the crack length, $\mathrm{Z}$ is a function of the dimensionless ratio:

$$
Z=f\left(\frac{L}{d}\right),
$$

where $\mathrm{L}$ is the length of the crack. Denote the maximal value of this function by $\mathrm{Z}_{\max }$. No cracks can advance if the crack of maximal energy release rate is below the surface energy:

$$
\mathrm{Z}_{\text {max }} \mathrm{E} \varepsilon_{\mathrm{m}}^{2} \mathrm{~d}<2 \gamma .
$$

This condition defines a critical particle size,

$$
\mathrm{d}_{\text {critical }}=\frac{2 \gamma}{\mathrm{Z}_{\max } \mathrm{E} \varepsilon_{\mathrm{m}}^{2}} .
$$

When the particle is smaller than this critical size, no pre-existing cracks in the particle can advance.

The above approach has been used to analyze many systems, such as polycrystals ${ }^{25}$, composites $^{26}$, and thin films ${ }^{27}$. We now apply this approach to crystalline particles of LiFePO4. The crystal has the olivine structure (Fig.2). In the b direction of the crystal are tunnels, in which lithium ions diffuse. 9,11,12,28 The crystal has two phases: the lithium-rich phase noted as LiFePO4, and the lithium-poor phase noted as FePO4. The lattice constants of the LiFePO4 phase are $\mathrm{a}=10.33 \AA, \mathrm{b}=5.79 \AA$, and $\mathrm{c}=4.69 \AA$, while the lattice constants of the FePO4 phase are $\mathrm{a}=9.81 \AA, \mathrm{b}=5.79 \AA$, and $\mathrm{c}=4.78 \AA .{ }^{17}$ Consequently, upon absorbing lithium and changing phase, the crystal deforms by a state of triaxial strains:

$$
\varepsilon_{\mathrm{a}}=5.03 \%, \quad \varepsilon_{\mathrm{b}}=3.7 \%, \quad \varepsilon_{\mathrm{c}}=-1.9 \% .
$$

This state of strains is anisotropic. 
We analyzed the field of stress by using a commercial finite element package, ABAQUS. The strains associated with the phase transition were applied as if they were caused by thermal expansion. The crack tip was densely meshed, with the smallest element around the crack tip being $10^{-4}$ times of the crack length. Orthotropic material model was used and the elastic moduli used here were calculated by using a first-principles method ${ }^{29}$. Experimental observations of plate-like and sphere-like particles were reported. ${ }^{11}$ We represented a plate-like particle by a plan stress model, and a sphere-like particle by plane strain model. The mismatch strains (6) were prescribed as anisotropic thermal strains in ABAQUS. The J-integral was used to calculate energy release rate.

Fig. 3 illustrates a plate-like particle lying in the ac-plane, with the phase boundary in the bc-plane. We consider a crack on this plane. The plate is taken to deform under the plane stress conditions. The calculated energy release rate is plotted as a function of the crack length. When the crack is very short, the elastic energy in the particle does not change appreciably when the length of the crack changes, and the energy release rate is small. When the crack is very long, the elastic energy in the particle is nearly fully relaxed, and the energy release rate is also small. The energy release rate maximizes when the crack is of an intermediate length, giving $\mathrm{Z}_{\max }=0.0363$. In this case, $\mathrm{c}_{11}=166.5 \mathrm{GPa}$ in the stiffness matrix of $\mathrm{FePO} 4^{29}$ and $\varepsilon_{\mathrm{c}}=-1.9 \%$ were used for normalization. The surface energy of the bc-plane from the first-principles calculation is $\gamma_{\mathrm{bc}}=0.66 \mathrm{~J} / \mathrm{m}^{2} .{ }^{29}$ From Eq. (5), the critical size of the plate-like particle is estimated to be $d_{\text {critical }}=605 \mathrm{~nm}$.

Fig. 4 illustrates particles of a sphere-like shape. Experimental observations have shown that the phase boundary is on the ac-plane, and the crack can be on the two cleavage planes, bcplane or ac-plane. ${ }^{11}$ In the finite-element calculation, the sphere-like particle is taken to deform under the plane strain conditions. Fig. 4a plots the calculated energy release rate as a function of the crack length for a crack on the bc-plane, giving $Z_{\max }=0.0065$. Fig. $4 \mathrm{~b}$ plots the calculated 
results for a crack on the ac-plane, giving $Z_{\max }=0.0524$. Consequently, the critical particle size should be determined by the crack on the ac-plane. In this case, $c_{11}=166.5 \mathrm{GPa}$ in the stiffness matrix of FePO4 and $\varepsilon_{\mathrm{a}}=5.03 \%$ were used for normalization. Surface energy of the ac-plane from the first-principles calculations is $\gamma_{\text {ac }}=0.64 \mathrm{~J} / \mathrm{m}^{2} .29$ The critical particle size for spherelike particle calculated from Eq. (5) is $d_{\text {critical }}=58 \mathrm{~nm}$.

Experimental observations of cracked particles reported in the literature are limited. Cracks were reported in a plate-like particle of dimensions $4 \times 2 \times 0.2 \mu \mathrm{m} .{ }^{11,12}$ Cracks were also reported in sphere-like particles of size $300-600 \mathrm{~nm}$ and $200 \mathrm{~nm} \cdot{ }^{9,11}$ When particle size is smaller than $30 \mathrm{~nm}$, no crack was reported. ${ }^{30}$ These experimental observations are consistent with the critical particle size calculated in this paper. To further test the accuracy of this model, more experimental data on cracks in particles of different sizes and shapes are needed. In addition, in calculating critical particle sizes, we have used the elastic moduli and surfaces energies determined by first-principles calculations ${ }^{29}$. It is well-known that the critical energy release rate is typically larger than two times the surface energy. ${ }^{24}$ Both the elastic moduli and critical energy release rates should be ascertained by future experiments.

In summery, we have applied fracture mechanics to study cracking in crystalline particles of LiFePO4 caused by the mismatch between different phases. We estimate the maximal energy release rate among various cracks. When the maximal energy release rate is below the critical value, no crack in the particle can advance. This approach enables us to calculate the critical particle size to avert cracking. It is hoped that more experimental observations will be made available to ascertain the theoretical calculation.

The authors are grateful to the support by DARPA through a contract on Programmable Matter, and by NSF through a grant on Soft Active Materials. 


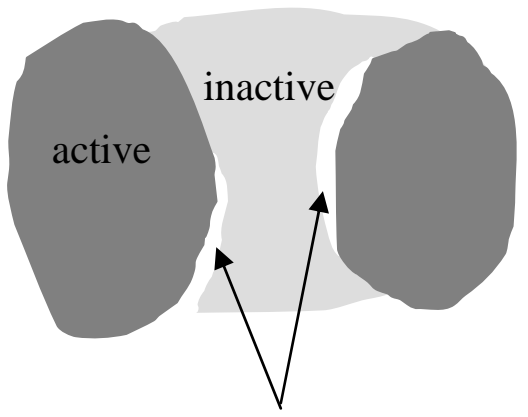

debond
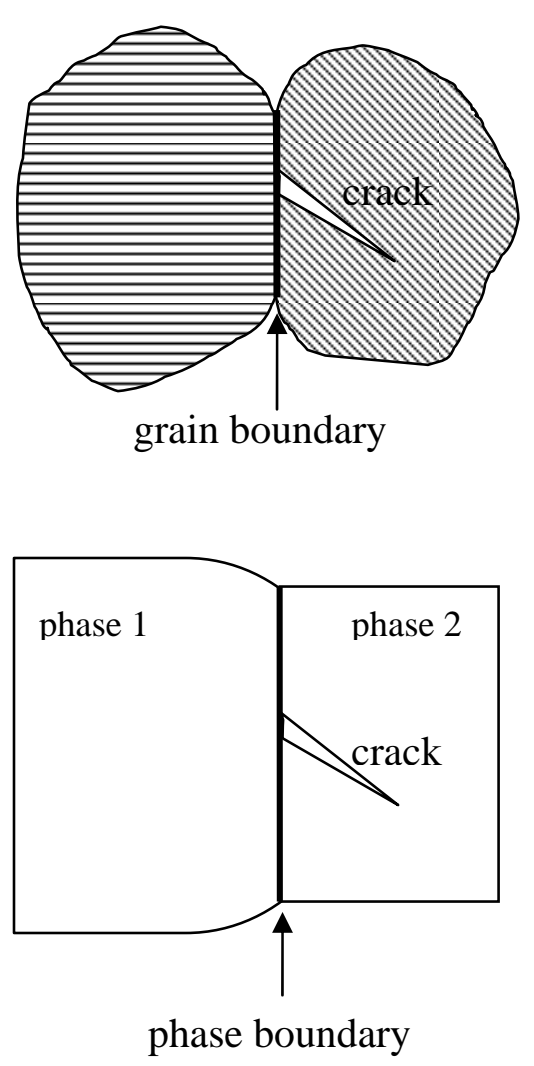

FIG.1. Insertion-induced deformation may be constrained by the mismatch between active and inactive materials, between grains of different orientations, and between phases of different concentrations of lithium. The constrained deformation leads to stresses, which may cause the electrode to crack. 


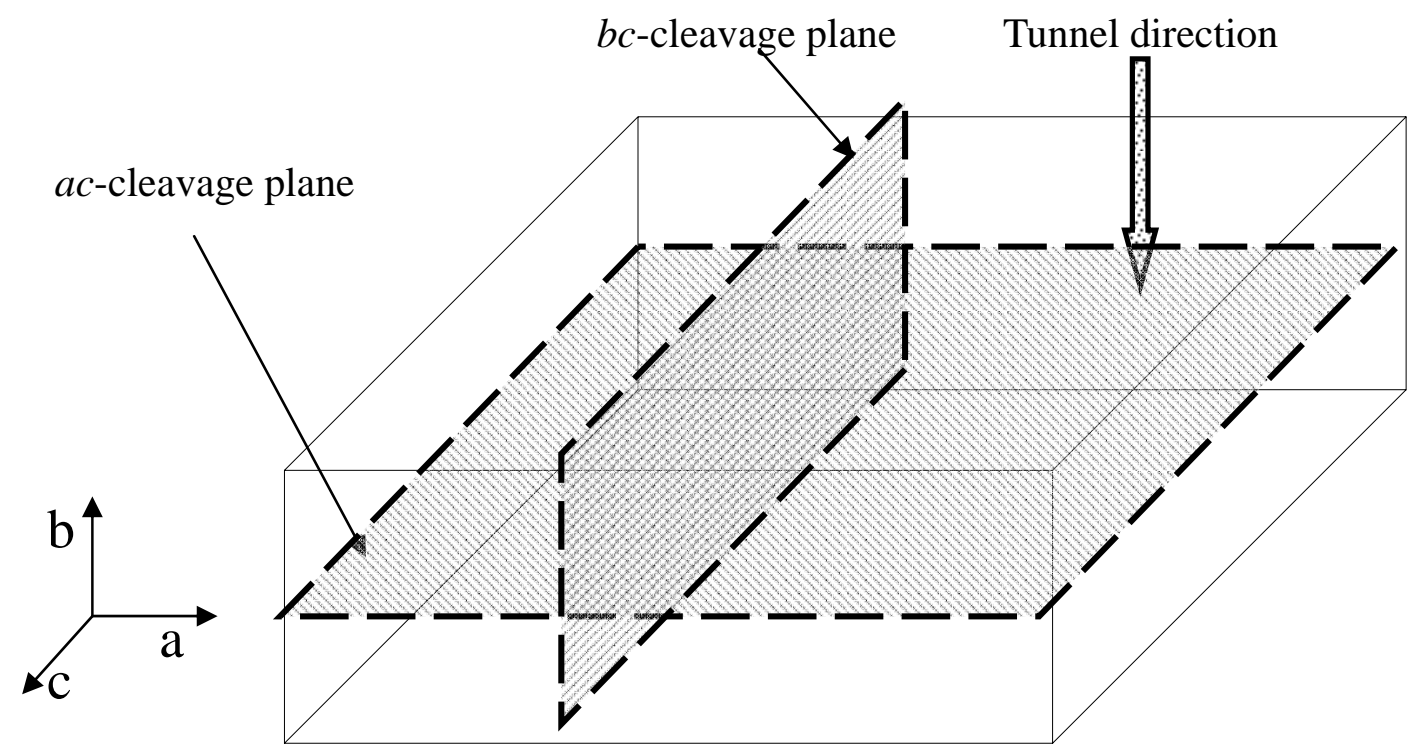

FIG.2. In a crystal of LiFePO4, lithium atoms diffuse along tunnels in direction b, and cleavage may occur on the bc and ac planes. 


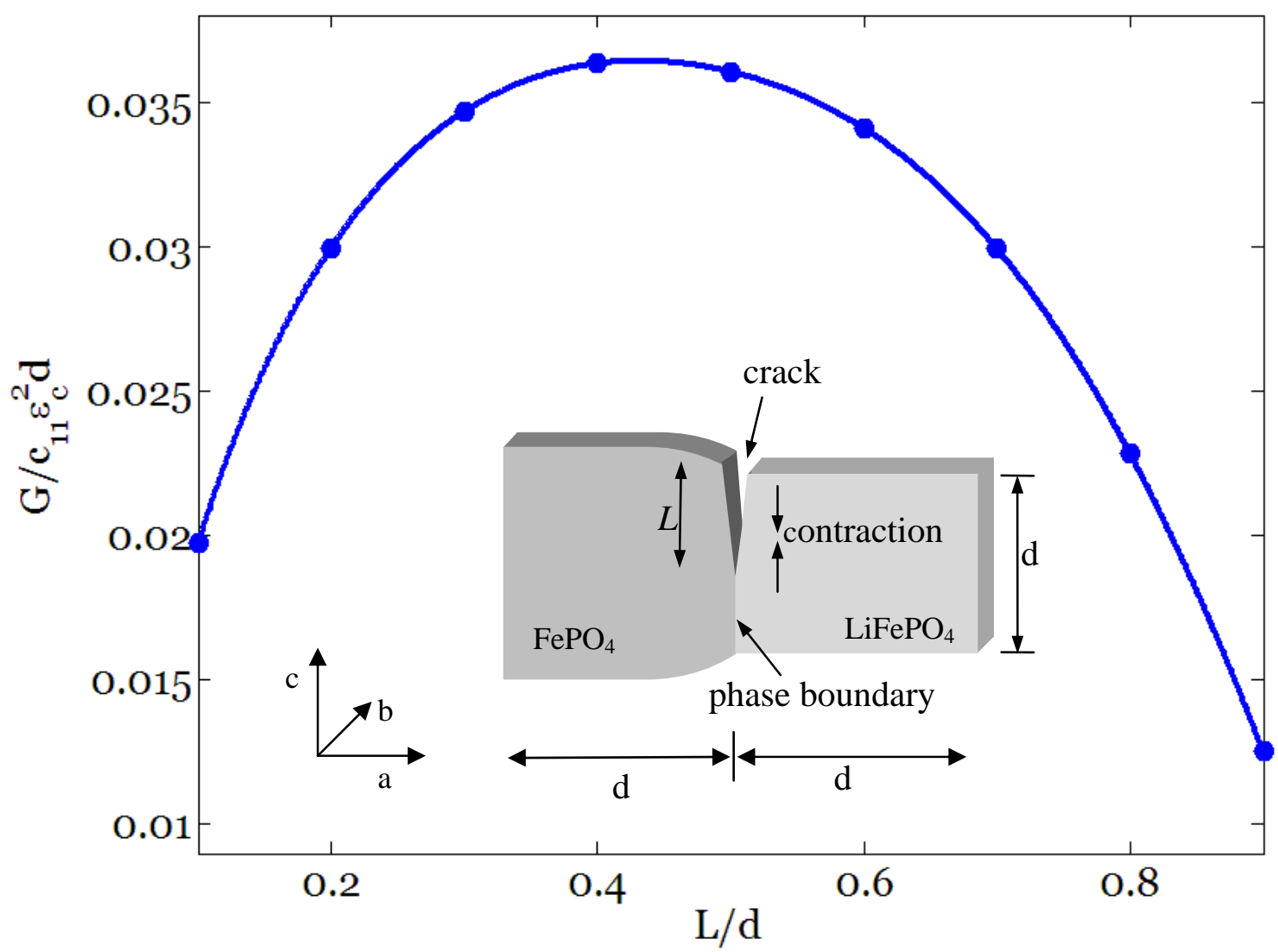

FIG.3. Energy release rate for a crack on the phase boundary in a plate-like LiFePO4 particle. 


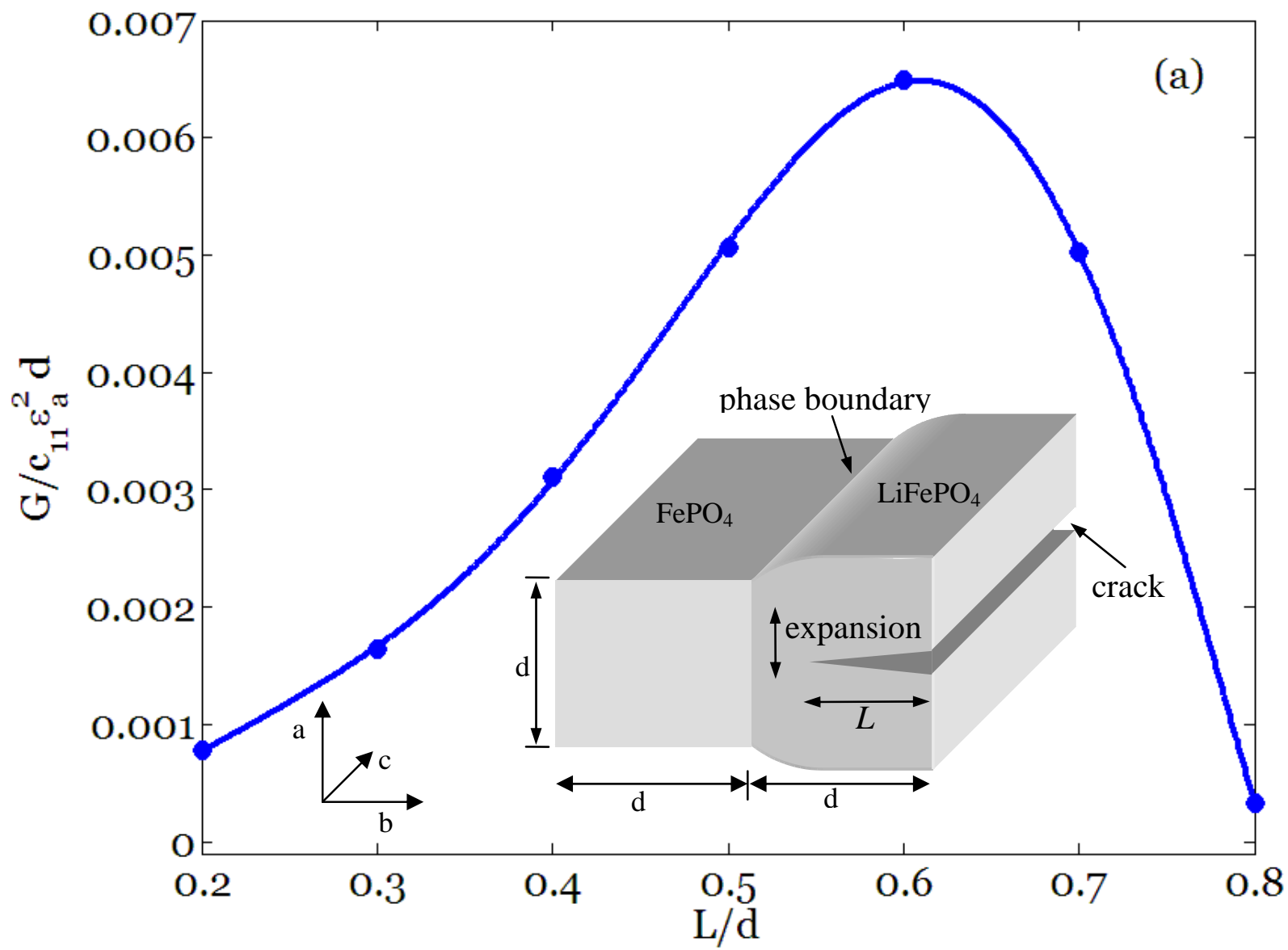




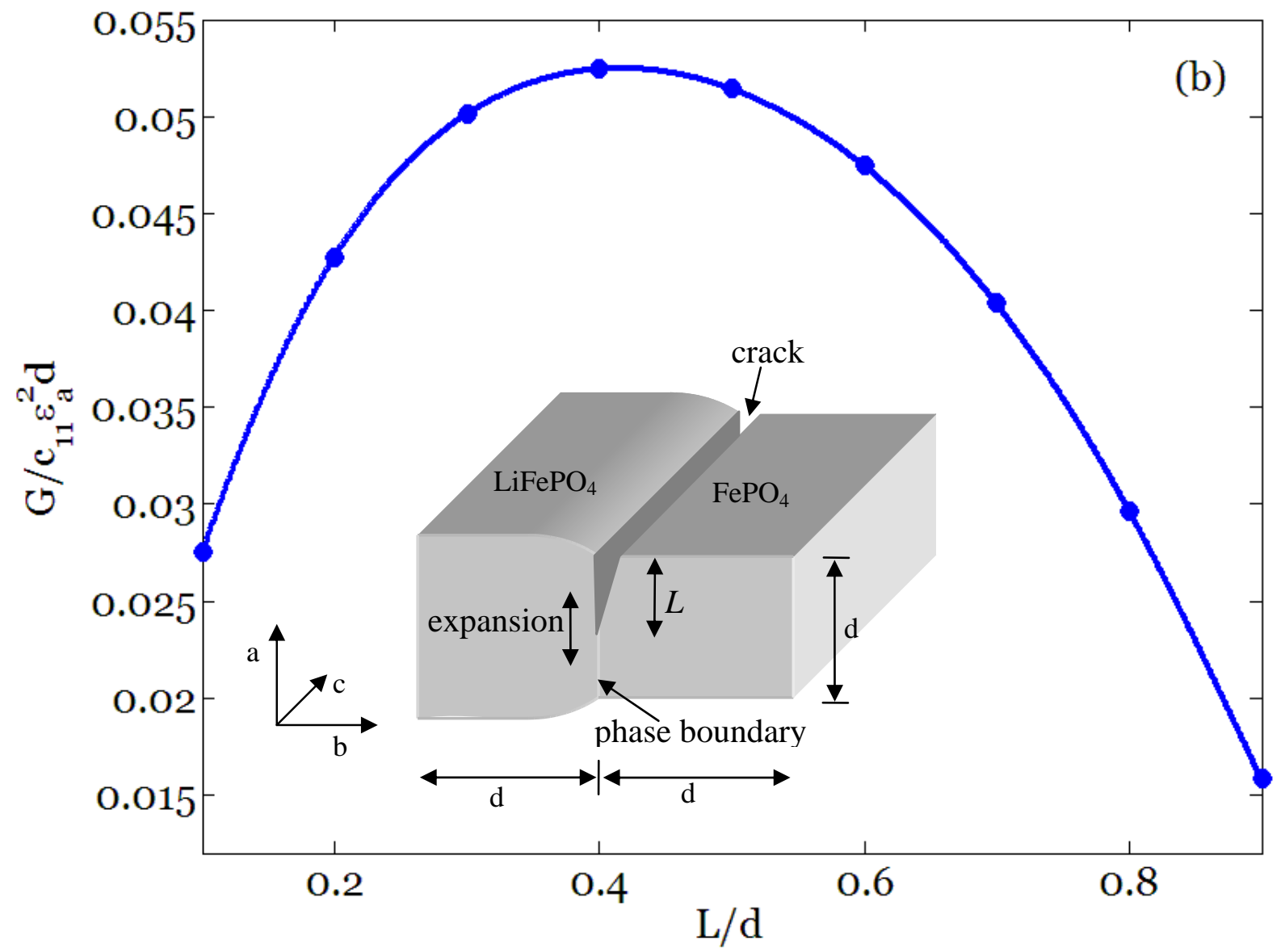

FIG. 4. In a sphere-like LiFePO4 particle, energy release rate of (a) a crack in a phase and, (b) a crack on the phase boundary. 


\section{References}

1. G. A. Nazri, and G. Pistoia, Lithium Batteries, Springer (2003).

2. Department of Energy, Basic Research Needs to Assure a Secure Energy Future (2003).

3. Department of Energy, Basic Research Needs for Electrical Energy Storage (2007).

4. M. Broussely, and G. Pistoia, Industrial Applications of Batteries, Elsevier, Amsterdam (2007).

5. P. G. Bruce, Solid State Electrochemistry, Cambridge University Press (1995).

6. R. A. Huggins, Advanced Batteries, Springer (2009).

7. J. Go, and I. Pyun,2003. J . Electrochem. Soc. 150, A1037 (2003).

8. Y. Itou, and Y. Ukyo, J . Power Sourc. 146, 39 (2005).

9. D. Wang, X. Wu, Z. Wang, and L. Chen, J . Power Sourc. 140, 125 (2005).

10. K. E. Aifantis, and S. A. Hackney, J . Mech. Behav. Mater. 14, 413 (2003).

11. H. Gabrisch, J. Wilcox, and M. M. Doeff, Electrochem. Solid-State Lett. 11, A25 (2008).

12. G. Chen, X. Song, and T. J . Richardson, Electrochem. Solid-State Lett. 9, A295 (2006).

13. R. A. Huggins and W. D. Nix, Ionics 6, 57 (2000).

14. K. E. Aifantis, S. A. Hackney, and J . P. Dempsey, J . Power Sourc. 165, 874 (2007).

15. X. C. Zhang, W. Shyy, and A. M. Sastry, J . Electrochem. Soc. 154, A910 (2007).

16. J . Christensen, and J . Newman, J . Electrochem. Soc. 153, A1019 (2005).

17. A. K. Padhi, K. S. Nanjundaswamy, and J . B. Goodenough, J. Electrochem. Soc. 144, 1188 (1997)

18. K. Zaghib, J. Shim, A. Guerfi, P. Charest, and K. Striebel, Electrochem. Solid-State Lett. 8, A207 (2005).

19. S.Y. Chung, J. T. Bloking, and Y. M. Chiang, Nat. Mater. 1, 123 (2002).

20. S. P. Herle, B. Ellis, N. Coombs, and L. F. Nazar, Nat. Mater. 3, 147 (2004).

21. F. Croce, A. D. Epifanio, J. Hassoun, A. Deptula, T. Olczac, and B. Scrosati, Electrochem. Solid-State Lett. 5, A47 (2002).

22. A. Yamada, S. C. Chung, and K. Hinokuma, J. Electrochem. Soc. 148, A224 (2001).

23. C. Delacourt, P. Poizot, S. Levasseur and C. Masquelier, Electrochem. Solid-State Lett. 9, A352 (2006).

24. B. Lawn, Fracture of Brittle Solids, Cambridge University Press (1993).

25. A. G. Evans, Acta Metall. 26, 1845 (1978).

26. T. C. Lu, J. Yang, Z. Suo, A. G. Evans, R. Hecht and R. Mehrabian, Acta Metall. Mater. 39, 1883 (1991).

27. J. W. Hutchinson and Z. Suo, Adv. Appl. Mech. 29, 63 (1992).

28. C. Ouyang, S. Shi, Z. Wang, X. Huang and L. Chen, Phys. Rev. B 69, 104303 (2004).

29. T. Maxisch and G. Ceder, Phys. Rev. B 73, 174112 (2006).

30. K. Hsu, S. Tsay, and B. Hwang, J . Mater. Chem. 14, 2690 (2004). 